\title{
RIEMANN BOUNDARY-VALUE PROBLEM ON CASSINI SPIRALS
}

\begin{abstract}
Recently, a number of papers has been published concerning the Riemann boundary-value problems on spiral-like arcs. In all these works, the spiral has the shape close to concentric circles, i. e., with equal rates of torsion in all directions. This paper is the first study of this problem on spirals with oval turns.
\end{abstract}

Key words: Rimann boundary-value problem, non-rectifiable curve, spirals, strong torsion, Cassini ovals.

\section{Mathematical Subject Classification: 30E20}

1. Introduction. We consider the Riemann boundary-value problem on an arc $\Gamma$. Its statement is well known. Let $\Gamma$ be a directed simple Jordan arc beginning at a point $a_{1}$ and ending at a point $a_{2}$. We seek for all holomorphic in $\overline{\mathbb{C}} \backslash \Gamma$ functions $\Phi(z)$, such that

(RP1) they have limit values $\Phi^{ \pm}(t)$ from the left and from the right at any point $t \in \Gamma^{\prime}:=\Gamma \backslash\left\{a_{1,2}\right\}$;

(RP2) these boundary values are connected by relation

$$
\Phi^{+}(t)=G(t) \Phi^{-}(t)+g(t)
$$

where $G(t)$ and $g(t)$ are given functions;

$(\mathrm{RP} 3) \Phi(\infty)=0$;

(RP4) the desired functions $\Phi(z)$ satisfy the following bounds near the end-points $a_{1,2}$ :

$$
\Phi(z)=O\left(\left|z-a_{j}\right|^{-\gamma_{j}}\right), \quad 0 \leqslant \gamma_{j}<1, \quad j=1,2 .
$$

There exists a great body of papers on this problem. In the classical works (see, for instance, [1-3]) the arc $\Gamma$ is assumed piecewise-smooth. 
Later, the Riemann problem was solved on non-rectifiable arcs [4-6]. However, these papers contain additional restrictions on behavior of the $\operatorname{arc} \Gamma$ at its end-points. In order to describe these restrictions, we introduce the so called logarithmic kernel of the arc $\Gamma$ :

$$
K_{\Gamma}(z):=\frac{1}{2 \pi i} \ln \frac{z-a_{2}}{z-a_{1}} .
$$

It is a single-valued branch of logarithm, holomorphic in $\overline{\mathbb{C}} \backslash \Gamma$, and uniquely determined by condition $K_{\Gamma}(\infty)=0$. Clearly,

$$
K_{\Gamma}(z)=A_{\Gamma}(z)+\frac{1}{2 \pi i} \ln \left|\frac{z-a_{2}}{z-a_{1}}\right|,
$$

where $A_{\Gamma}(z)$ is a real-valued function. Generally speaking, singularities of arbitrarily high orders at the points $a_{1,2}$ are allowed. The Riemann boundary-value problem on non-rectifiable arcs is solved in [4], [6] under one of the following assumptions:

- if $G(t) \equiv 1$ (the so called jump problem) and $K_{\Gamma}(z)$ is square integrable near the points $a_{1,2}$;

- if $g(t) \equiv 0$ (the homogeneous problem) and $K_{\Gamma}(z)=O\left(-\ln \left|z-a_{j}\right|\right)$ near the points $a_{j}, j=1,2$;

- if the limits

$$
\lim _{z \rightarrow a_{j}} \frac{K_{\Gamma}(z)}{\ln \left|z-a_{j}\right|}, \quad j=1,2 .
$$

exist.

Recently, two articles [7], [8] have been published; they deal with the Riemann problems on arcs with singularities of logarithmic kernels of higher orders. The arc is called spiral of strong torsion if its logarithmic kernel is not integrable near one of its end-points. The solvability of the Riemann boundary-value problem on such arcs essentially depends on their shapes at the ends. In this paper, we study the Riemann boundaryvalue problem on spirals of strong torsion with Cassini oval-like turns.

2. Cassini spirals. Cassini ovals ${ }^{1}$ are domains determined on the complex plane by the relation

$$
C(p, q)=\left\{z:\left|z^{2}-q^{2}\right|<p^{2}\right\}, \quad p>q>0 .
$$

\footnotetext{
${ }^{1}$ named after the famous astronomer and mathematician Giovanni Domenico Cassini, 1625-1712
} 
In other words, it is the set of points such that the product of their distances from the focal points $\pm q$ is less than $p^{2}$.

Note that equation of the boundary of such oval can be written as

$$
\bar{z}^{2}=q^{2}+\frac{p^{4}}{z^{2}-q^{2}} .
$$

Let $C\left(p_{1,2}, q_{1,2}\right)$ be two Cassini ovals. Elementary transformations show that if $q_{1}<q_{2}$ and $q_{2}^{2}-q_{1}^{2}<p_{2}^{2}-p_{1}^{2}$, then $C\left(p_{1}, q_{1}\right) \subset C\left(p_{2}, q_{2}\right)$.

Fix two decreasing sequences of positive values $\mathbf{p}=\left\{p_{1}, p_{2}, \ldots\right\}$ and $\mathbf{q}=\left\{q_{1}, q_{2}, \ldots\right\}$, such that $q_{n+1}^{2}-q_{n}^{2}<p_{n+1}^{2}-p_{n}^{2}$ for any positive integer $n$ and $\lim _{n \rightarrow \infty} p_{n}=\lim _{n \rightarrow \infty} q_{n}=0$, and consider the sequence of consecutively embedded Cassini ovals $C_{n}=C\left(p_{n}, q_{n}\right), n=1,2, \ldots$, such that $\bigcap_{n=1}^{\infty} C_{n}=\{0\}$. Denote $R_{n}:=C_{n} \backslash C_{n+1}$. Let $x_{n}$ be the point of intersection between boundary of the oval $C_{n}$ with the positive ray of the real axis $R_{+}=[0,+\infty)$.

In each domain $R_{n}$, we consider Jordan arcs $\gamma_{n}$ beginning at the point $x_{n+1}$ and ending at $x_{n}$. The domain $\Delta_{n}$ to the left of the arc (respectively, to the right) has the boundary $\gamma_{n} \cup\left[x_{n+1}, x_{n}\right]$. Let the arcs be such that this domain contains the oval $C_{n+1}$. The union of these arcs and the point 0

$$
\Gamma=\left(\cup_{n=0}^{\infty} \gamma_{n}\right) \cup\{0\}
$$

is called the left (respectively, right) Cassini spiral. It is directed from the point 0 to the point $x_{1}$. We define the signature of the spiral as the value $\sigma_{\Gamma}$ that equals +1 or -1 for the left and right spiral, correspondingly.

If $\Gamma$ is a Cassini spiral, then the argument of its logarithmic kernel has the asymptotic estimations

$$
\begin{gathered}
A_{\Gamma}(z)=\sigma_{\Gamma} \sum_{n=1}^{+\infty} \chi_{n}(z)+O(1), \quad z \rightarrow 0, \\
A_{\Gamma}(z)=O(1), \quad z \rightarrow x_{1},
\end{gathered}
$$

where $\chi_{n}$ is the characteristic function of the oval $C_{n}$.

The oval $C(p, q)$ satisfies the inclusion

$$
\left\{z:|z|<\sqrt{p^{2}-q^{2}}\right\} \subset C(p, q) \subset\left\{z:|z|<\sqrt{p^{2}+q^{2}}\right\} .
$$

Hence, if

$$
\sum_{n=1}^{+\infty}\left(p_{n}^{2}-q_{n}^{2}\right)=\infty
$$


then the Cassini spiral has strong torsion at the origin. For instance, this concerns the spiral with $q_{n}=n^{-\alpha}, p_{n}=k q_{n}, 0<\alpha \leqslant 1 / 2, k>1$.

If $\operatorname{arc} \gamma_{n}$ is rectifiable, then its length $\left|\gamma_{n}\right|$ exceeds $2 \pi \sqrt{p_{n}^{2}-q_{n}^{2}}$, and, under condition (2), the total length of $\Gamma$ is infinite.

In what follows, we assume, for the sake of brevity, that $\Gamma$ satisfies the so called Luzin condition, i. e., any portion of $\Gamma$ contains a rectifiable subarc. As noted by N. I. Luzin, a curve with this property is erasable in the class of continuous functions (see, for instance, [9]). Clearly, a curve satisfying Luzin condition has null plane measure.

3. The jump problem. We consider first the jump problem, i.e., the problem (RP1) - (RP4) with $G \equiv 1$. In the piecewise smooth case, its solution is given by the Cauchy type integral, but Cassini spirals of strong torsion cannot be rectifiable, and this integral diverges. In order to build a solution, we use the following functional classes.

As customary, the Lipschitz space Lip $\Gamma$ consists of functions $f$ defined on $\Gamma$ and satisfying the condition

$$
\sup \left\{\frac{\left|f(t)-f\left(t^{\prime}\right)\right|}{\left|t-t^{\prime}\right|}: t, t^{\prime} \in \Gamma, t \neq t^{\prime}\right\}:=\Lambda(f)<+\infty .
$$

The Taylor space $T^{m}(\Gamma)$ consists of functions $f$ representable as

$$
f(\tau)=P_{f}(\tau, \bar{\tau})+\tau^{m} f_{0}(\tau), \quad f_{0} \in \operatorname{Lip} \Gamma,
$$

where $P_{f}(\tau, \bar{\tau})$ is the Taylor polynomial of degree less than $m$.

We use its subspaces: $T_{0}^{m}(\Gamma)$ consists of functions $f \in T^{m}(\Gamma)$ with null Taylor polynomials; $T_{H}^{m}(\Gamma)$ consists of functions $f \in T^{m}(\Gamma)$ with holomorphic Taylor polynomials; $T_{E}^{m}(\Gamma)$ consists of functions $f \in T^{m}(\Gamma)$, such that the Taylor polynomial $P_{f}(\tau, \bar{\tau})$ contains only even degrees of $\bar{\tau}$.

Let a Cassini spiral $\Gamma$ satisfy the bound

$$
K_{\Gamma}(z)=O\left(|z|^{-m}\right), \quad z \rightarrow 0,
$$

and $g \in T_{0}^{m}(\Gamma)$. Consider the function

$$
U(z):=K_{\Gamma}(z) z^{m} \mathcal{E} g_{0}(z) \psi(z),
$$

where $\mathcal{E}$ is the Whitney extension operator (see, for instance, [10]), and $\psi(z)$ is a smooth function with compact support; it equals unit in a neighborhood of $\Gamma$. The function

$$
\Phi_{0}(z):=U(z)-\frac{1}{2 \pi i} \iint_{\mathbb{C}} \frac{\partial U}{\partial \bar{\zeta}} \frac{d \zeta d \bar{\zeta}}{\zeta-z}
$$


is holomorphic in $\mathbb{C} \backslash \Gamma$, vanishes at the infinity, has a jump $g$ on $\Gamma$, and satisfies the bounds

$$
\Phi_{0}(z)=O(1), z \rightarrow 0, \quad \Phi_{0}(z)=g\left(x_{1}\right) K_{\gamma}(z), z \rightarrow x_{1}
$$

at the end-points of the arc. Hence, it is a solution of the jump in this case.

Then, consider the case $g \in T_{H}^{m}(\Gamma)$ and the function

$$
\Phi_{1}(z):=\Phi_{0}(z)+P_{g}(z) K_{\Gamma}(z)-Q(z),
$$

where $Q(z)$ is a holomorphic polynomial, such that the function $\Phi_{1}$ vanishes at the infinity. This polynomial exists and is unique. The function $\Phi_{1}$ satisfies conditions (RP1)-(RP3), but the bounds (4) here turn into the asymptotic formulae

$$
\Phi_{1}(z)=P_{g}(z) K_{\Gamma}(z)+O(1), z \rightarrow 0, \quad \Phi_{1}(z)=g\left(x_{1}\right) K_{\gamma}(z), z \rightarrow x_{1} .
$$

Hence, the problem with this jump becomes resolvable if we replace the condition (RP4) by the bounds

$$
\Phi(z)=O\left(z^{-m}\right), z \rightarrow 0, \quad \Phi(z)=O\left(\left|z-x_{1}\right|^{-\gamma}\right), z \rightarrow x_{1}, 0 \leqslant \gamma<1,
$$

which we call condition (RP5). The general solution can be written as

$$
\Phi(z)=\Phi_{1}(z)+z^{-m} P(z)
$$

where $P(z)$ is an arbitrary polynomial of degree less than $m$.

Let $g \in T_{E}^{m}(\Gamma)$, i. e.,

$$
g(t)=t^{m} g_{0}(t)+P_{g}(t, \bar{t}), \quad P_{g}(t, \bar{t})=\sum_{\alpha, \beta \geqslant 0}^{\alpha+2 \beta<m} c_{\alpha, 2 \beta} t^{\alpha} \bar{t}^{2 \beta},
$$

where $g_{0} \in \operatorname{Lip} \Gamma, \alpha$ and $\beta$ are non-negative integers, and $c_{\alpha, \beta}$ are constants. The function $\Phi_{0}$ has a jump $t^{m} g_{0}(t)$. It remains to construct a function with the jump $P_{g}(t, \bar{t})$. Connect the end-points of the spiral $\Gamma$ with the segment $I:=\left[0, a_{1}\right]$ and consider three jump problems:

$$
\begin{gathered}
\Phi_{21}^{+}(\tau)-\Phi_{21}^{-}(\tau)=\sigma P(\tau, \bar{\tau}), \quad \tau \in \cup_{n=1}^{\infty} \partial \Delta_{n}^{\prime} \\
\Phi_{22}^{+}(\tau)-\Phi_{22}^{-}(\tau)=P_{g}(\tau, \bar{\tau}), \quad \tau \in I
\end{gathered}
$$




$$
\Phi_{3}^{+}(\tau)-\Phi_{3}^{-}(\tau)=\sigma P_{g}(\tau, \bar{\tau}), \quad \tau \in \cup_{n=1}^{\infty} C_{n} .
$$

The contour of the first problem is the union of boundaries of the domains $\Delta_{n}^{\prime}:=\Delta_{n} \backslash C_{n+1}$. As these domains do not overlap, one of the solutions is the generalized Cauchy-type integral

$$
\Phi_{1}(z):=\sigma P(z, \bar{z})-\frac{\sigma}{2 \pi i} \iint_{\cup_{n=1}^{\infty} \partial \Delta_{n}^{\prime}} \frac{\partial P}{\partial \bar{w}} \frac{d w d \bar{w}}{w-z}
$$

which is bounded in the whole complex plane and vanishes at the infinity.

The solution of the second problem is the customary integral of the Cauchy type:

$$
\Phi_{2}(z)=\frac{1}{2 \pi i} \int_{I} \frac{P(\tau, \bar{\tau}) d \tau}{\tau-z}
$$

with well-known properties.

Finally, the contour of the third problem is the infinite set of boundaries of the Cassini ovals $C_{n}$. By virtue of the formula (1), the sum

$$
S(z):=\sum_{n=1}^{\infty} \chi_{n}(z) \sum_{\alpha, \beta \geqslant 0}^{\alpha+2 \beta<m} c_{\alpha, 2 \beta} z^{\alpha}\left(q_{n}^{2}+\frac{p_{n}^{4}}{z^{2}-q_{n}^{2}}\right)^{\beta}
$$

satisfies the boundary-value condition of the jump problem. It has poles at points $\pm q_{n}$, but we can "erase" them by using the Mittag-Leffler theorem (see, for instance, [11]). According to this theorem, there exists a function $M(z)$, meromorphic in $\overline{\mathbb{C}} \backslash\{0\}$, such that it has poles at the points $\pm q_{n}$ only, and its main parts at the poles coincide with the main parts of $S(z)$. Then the sum

$$
\Phi_{*}(z)=\Phi_{0}(z)+\Phi_{1}(z)+\Phi_{2}(z)+S(z)-M(z)
$$

satisfies the conditions (RP1)-(RP3). Its asymptotic at the origin is unknown.

Thus, we have proved the following

Theorem 1. Let the Cassini spiral of strong torsion $\Gamma$ satisfy Luzin's condition and the bound (3). Then the following results are valid:

If $g \in T_{0}^{m}(\Gamma)$, then the jump problem (RP1)-(RP4) has a unique solution. 
If $g \in T_{H}^{m}(\Gamma)$, then the jump problem (RP1), (RP1), (RP3), (RP5) is resolvable, and its general solution is (5), where $P(z)$ is an arbitrary polynomial of degree less than $m$.

If $g \in T_{E}^{m}(\Gamma)$, then the jump problem $(R P 1)-(R P 3)$ is resolvable, and its general solution is $\Phi_{*}(z)+F\left(z^{-1}\right)$, where $F(w)$ is an arbitrary entire function, such that $F(0)=0$.

4. Homogeneous problem. We consider here the homogeneous Riemann boundary-value problem on a Cassini spiral of strong torsion, putting $g \equiv 0, G(t)=\exp f(t), f \in T^{m}(\Gamma)$.

Assume first that $f \in T_{0}^{m}(\Gamma)$. Let $\phi_{0}(z)$ be a solution of the jump problem with the jump $f(t)$, i. e.,

$$
\phi(z)=u(z)-\frac{1}{2 \pi i} \iint_{\mathbb{C}} \frac{\partial u}{\partial \bar{\zeta}} \frac{d \zeta d \bar{\zeta}}{\zeta-z},
$$

where $u(z):=K_{\Gamma}(z) z^{m} \mathcal{E} f_{0}(z) \psi(z)$. Denote $X_{0}(z)=\exp \phi(z)$. Obviously,

$$
X_{0}^{+}(t)=G(t) X_{0}^{-}(t), \quad t \in \Gamma^{\prime} .
$$

This function is bounded near the origin, and near end-point of $\Gamma$ we have

$$
\ln \left|X_{0}(z)\right|=\operatorname{Re} f\left(x_{1}\right) K_{\Gamma}(z)+O(1) .
$$

We put $f\left(x_{1}\right)=a+i b$, where $a$ and $b$ are real. Then

$$
\ln \left|X_{0}(z)\right|=(2 \pi)^{-1} b \ln \left|z-x_{1}\right|+O(1), \quad z \rightarrow x_{1},
$$

because the argument $A_{\Gamma}$ is bounded near $x_{1}$. Denote by $\varkappa$ the entire part of $(2 \pi)^{-1} b$, and put $X(z)=\left(z-x_{1}\right)^{-\varkappa} X_{0}(z)$. Clearly, for $\varkappa \leqslant 0$ this homogeneous Riemann problem has the null solution only, and for $\varkappa>0$ its general solution is $\Phi(z)=X(z) P(z)$, where $P$ is an arbitrary polynomial of degree less than $\varkappa$.

Now we consider the case $f \in T_{H}^{m}(\Gamma)$. The corresponding solution of the jump problem is

$$
\phi_{1}(z):=\phi_{0}(z)+P_{f}(z) K_{\Gamma}(z)-Q(z)
$$

where $Q(z)$ is the polynomial, such that $\phi_{1}$ vanishes at the infinity point. We put $X_{0}(z)=\exp \phi_{1}(z)$. Clearly, it satisfies the boundary-value condition (6) and the estimate (). Therefore, the function 
$X(z):=\left(z-x_{1}\right)^{-\varkappa} X_{0}(z)$ has the asymptotic $X(z) \asymp\left(z-x_{1}\right)^{-\gamma}, 0 \leqslant \gamma<1$, at the point $x_{1}$. Near the origin, we have

$$
\ln |X(z)|=A_{\Gamma}(z) \operatorname{Re} P_{f}(z)+(2 \pi)^{-1} \ln |z| \operatorname{Im} P_{f}(z)+O(1) .
$$

Let $\Phi(z)$ be a solution of the homogeneous problem (RP1) - (RP4). Then the ratio $F(z)=\Phi(z) / X_{0}(z)$ is holomorphic in $\overline{\mathbb{C}} \backslash\{0, \infty\}$, has order $\varkappa-1$ at $\infty$, and satisfies the bound

$$
|F(z)| \asymp \exp \left(-A_{\Gamma}(z) \operatorname{Re} P_{f}(z)\right)
$$

near the origin. Here we restrict ourselves by the case where the polynomial $P_{f}(z)$ has null of positive order $k<m$ at the origin, i. e.,

$$
P_{f}(z)=\sum_{n=k}^{m-1} c_{n} z^{n}, \quad c_{k} \neq 0 .
$$

Then $A_{\Gamma}(z) \operatorname{Re} P_{f}(z)=O\left(\mid z^{k-m}\right)$. On the other hand, the function $F(z)$ is bounded on the set $Z:=\left\{z: \operatorname{Re} P_{f}(z)=0\right\}$. This set contains $2 k$ arcs beginning at the origin, and these arcs divide a neighborhood of the origin on curvilinear sectors with angles $\pi / k$ at the origin. If $k>m-k$, then $F$ is holomorphic at the origin due to the Phragmen-Lindelöf principle (see, for instance, [11]), and, consequently, $F \equiv 0$.

The results of the previous section enable us to solve also the homogeneous Riemann problem on a Cassini spiral for $f \in T_{E}^{m}(\Gamma)$, but we do not have a bound for its solution at the origin. In other words, the obtained solution satisfies the conditions (R1) - (R3) only. Thus, the following theorem is proved:

Theorem 2. Let Cassini spiral of strong torsion $\Gamma$ satisfy Luzin's condition and the bound (3). Then the following results concerning the homogeneous Riemann problem are valid:

If $G=\exp f, f \in T_{0}^{m}(\Gamma)$, then the homogeneous problem $(R P 1)-(R P 4)$ for $\varkappa>0$ has a general solution $\Phi(z)=X(z) P(z)$, where $P$ is an arbitrary polynomial of degree less than $\varkappa$, and it has the null solution only for $\varkappa \leqslant 0$.

If $G=\exp f, f \in T_{H}^{m}(\Gamma)$ and the polynomial $P_{f}(z)$ has a root of order $k$ at the origin, $m>k>m / 2$, then the homogeneous problem (RP1)-(RP4) has the null solution only.

If $G=\exp f, f \in T_{E}^{m}(\Gamma)$, then the homogeneous problem $(R P 1)-(R P 3)$ is resolvable. 
5. Inhomogeneous problem. Consider now one case of the full Riemann boundary-value problem on a spiral. Let $G=\exp f, f \in T_{0}^{m}(\Gamma)$, and $g \in T_{0}^{m}(\Gamma)$. Then the customary factorization technique enables us to imply the following result:

Theorem 3. Let the Cassini spiral of strong torsion $\Gamma$ satisfy Luzin's condition and the bound (3), $G=\exp f, f \in T_{0}^{m}(\Gamma)$, and $g \in T_{0}^{m}(\Gamma)$. Then, for $\varkappa>0$, the inhomogeneous problem (RP1) - (RP4) has the general solution $\Phi(z)=\Psi(z)+X(z) P(z)$, where $\Psi(z)$ is its special solution and $P$ is an arbitrary polynomial of degree less than $\varkappa$. For $\varkappa \leqslant 0$, it has the unique solution under $-\varkappa$ solvability conditions.

We see that in this case the inhomogeneous Riemann boundary-value problem on Cassini spirals has the same solvability pattern as the problem on piecewise-smooth arcs [1-3]. Probably, its solution for boundary data in spaces $T_{E}^{m}(\Gamma)$ and $T_{H}^{m}(\Gamma)$ is more sophisticated.

Acknowledgment. This work was supported by the development program of the Scientific and Educational Mathematical Center of the Volga Federal District, agreement No. 075-02-2020-1478.

\section{References}

[1] Gakhov F. D. Boundary value problems. Nauka Publishers, 1977. English edition: Gakhov F. D. Boundary Value Problems. International Series of Monographs on Pure and Applied Mathematics Book, 1966, vol. 85 .

[2] Muskhelishvili N. I. Singular Integral Equations. Nauka Publishers, 1962 .

English edition: Muskhelishvili N. I. Singular Integral Equations. Boundary Problems of Function Theory and Their Application to Mathematical Physics. Dover Publications, 2013.

[3] Lu Jian-Ke. Boundary Value Problems for Analytic Functions. World Scientific, 1993.

[4] Kats B. A. Riemann boundary-value problem on a closed Jordan curve. Izvestiya vuzov. Matem., 1983, no. 4, pp. 68-80 (in Russian). English translation: Kats B. A. The Riemann problem on a closed Jordan curve. Soviet Math. (Iz. VUZ), 1983, vol. 27, no. 4, pp. 83-98.

[5] Kats B. A. Riemann problem on non-closed Jordan curve. Izvestiya vuzov. Matem., 1983, no 12, pp. 30-38 (in Russian). 
English translation: Kats B. A. The Riemann problem on an open Jordan curve. Soviet Math. (Iz. VUZ), 1983, vol. 27, no. 12, pp. 33-44.

[6] Kats B. A. On inhomogeneous Riemann problem on non-closed Jordan curve. Trudy semin. po kraevym zadacham, 1984, Kazan Univ. Publishers, vol. 21, pp. 87-93 (in Russian).

[7] Kats B. A., Katz D. B. Cauchy - Hadamard integral with applications. Monatschefte fur Matematik, 2019, vol. 189, no. 4, pp. 683-689.

DOI: https://doi.org/10.1007/s00605-019-01263-z

[8] Kats B. A. Zigzags and spirals in bounday-value problems. Izvestija vuzov. Matem. 2019, no. 7, pp. 39-47. (in Russian).

DOI: https://doi .org/10.26907/0021-3446-2019-7-39-47

English translation: Kats B. A. Zigzags and spirals in bounday-value problems. Russ Math., 2019, vol. 63, no. 7, pp. 33-40.

DOI: https://doi.org/10.3103/S1066369X19070041

[9] Markushevitch A. I. Selected chapters of theory of analytic functions. Nauka Publishers, 1976 (in Russian).

[10] Stein E. M. Singular integrals and differential properties of functions. Princeton University Press, 1970.

[11] Conway J. B. Functions of One Complex Variable I. (2nd ed.), Springer-Verlag New York, 1978.

DOI: https://doi.org/10.1007/978-1-4612-6313-5

Received November 19, 2020.

In revised form, January 20, 2021.

Accepted January 21, 2021.

Published online January 28, 2021.

Lyu Zhixine $^{a, b}$, Boris A. Kats ${ }^{a}$

a. Kazan Federal University, 18, Kremlevskaya st., Kazan, 420138, Russia

b. Tianjin University of Technology and Education(Tianjin China) Road Jingu No.5, Jinnan District, city Tianjin, China,

E-mail: Lyu ZhixineＩvzhixin0316@163.com Boris A. Kats katsboris877@gmail.com 